Version of Record: https:/www.sciencedirect.com/science/article/pii/S0515370019302009

Manuscript_0c1f64daa675c4880a51c5c80ab68c26

Dochead dossier

Sous-dochead L'essentiel sur les nouveaux médicaments à l'officine

\title{
Médicaments de la cardiologie, de l'infectiologie et de la dermatologie
}

\section{Jacques Buxeraud ${ }^{\mathrm{a}, *}$}

Professeur émérite des Universités

Sébastien Faure ${ }^{b}$

Professeur des Universités

${ }^{a}$ Faculté de pharmacie, 2 rue du Docteur-Marcland, 87025 Limoges cedex, France

'UFR santé, Département pharmacie, Université d'Angers, 16 boulevard Daviers, 49045 Angers, France

*Auteur correspondant.

Adresse e-mail : jacques.buxeraud@unilim.fr (J. Buxeraud).

Résumé

Les secteurs de la cardiologie et de la dermatologie se sont enrichis de nouveaux médicaments indiqués dans l'insuffisance cardiaque et le psoriasis en plaques. L'infectiologie bénéficie des plus grandes avancées avec la mise à disposition d'un nouvel antiviral indiqué dans le sida et d'antiviraux d'action directe qui permettent de traiter l'hépatite $C$.

(C) 2019

Mots clés - hépatite $C$; inhibiteur de néprilysine ; insuffisance cardiaque ; sida ; psoriasis

Summary à venir

(C) 2019

Keywords à venir 
En infectiologie, les médecins disposent dorénavant des antivirus hépatite $\mathrm{C}$ (VHC) pangénotypiques et d'un nouvel antiviral (Biktarvy ${ }^{\circledR}$ ) pour traiter le sida se composant de trois principes actifs. L'arrivée du premier inhibiteur de la néprilysine, le sacubitril associé à du valsartan (Entresto $\left.{ }^{\circledR}\right)$, permet, par ailleurs, de prendre en charge de façon originale I'insuffisance cardiaque. Enfin, le traitement du psoriasis s'est étoffé d'un anticorps monoclonal inhibiteur de l'interleukine 17 (IL-17), le brodalumab (Kyntheum ${ }^{\circledR}$ ).

\section{T1 Un médicament pour l'insuffisance cardiaque}

TEG1 Spécialité : Entresto ${ }^{\circledR}$ (sacubitril, valsartan), laboratoire Novartis Pharma SAS.

TEG1 Présentation : comprimé pelliculé à $24 \mathrm{mg} / 26 \mathrm{mg}$ (boîte de 28), 49 mg/51 mg (boîte de 56). et 97 mg/103 mg (boîte de 56).

TEG1 Prescription et délivrance : médicament en liste I.

TEG1 Remboursement Sécurité sociale à $65 \%$. Collectivités.

TEG1 Amélioration du service médical rendu (ASMR) : IV.

TEG1 Entresto ${ }^{\circledast}$ est indiqué dans le traitement de l'insuffisance cardiaque chronique symptomatique à fraction d'éjection réduite chez les patients adultes. Ce médicament possède un double mécanisme d'action grâce à ses deux composés.

TEG1 Le sacubitril, premier représentant d'une nouvelle classe thérapeutique, les inhibiteurs de néprilysine, agit via son métabolite actif, le LBQ657. La néprilysine a pour rôle de dégrader les peptides natriurétiques ${ }^{1}$. Ces peptides ne sont donc plus dégradés et entraînent une amélioration de l'état cardiaque grâce à divers effets pharmacologiques : vasodilatation, natriurèse et diurèse, augmentation de la filtration glomérulaire et du débit sanguin rénal, inhibition de la libération de rénine et d'aldostérone, diminution de l'activité sympathique, des effets anti-hypertrophique et antifibrotique.

TEG1 Le valsartan est un antagoniste des récepteurs de type 1 (AT1) de l'angiotensine II. II contre les effets néfastes cardiovasculaires et rénaux de l'angiotensine II en bloquant sélectivement les récepteurs AT1 ainsi que la libération d'aldostérone dépendante de l'angiotensine II, ce qui prévient l'activation continue du système rénine-angiotensine-aldostérone. Le valsartan a été choisi car sa propension à favoriser la survenue d'un angiœdème dans le cadre de l'association est acceptable $(0,5 \%)$ contrairement à celle d'un inhibiteur de l'enzyme de conversion (IEC) plus à risque [1-4].

Depuis début 2016, le traitement par sacubitril et valsartan était disponible à l'hôpital et en rétrocession dans le cadre du dispositif post-autorisation temporaire d'utilisation (ATU). 
TEG1 La posologie initiale recommandée de l'association sacubitril-valsartan est dans la plupart des cas d'un comprimé de $\mathbf{4 9} \mathbf{~ m g / 5 1 ~} \mathbf{m g}$ deux fois par jour, pendant ou en dehors des repas. Elle doit être doublée toutes les deux à quatre semaines jusqu'à la dose cible de $97 \mathrm{mg} / 103 \mathrm{mg}$ deux fois par jour, en fonction de la tolérance du patient. Toutefois, une diminution des doses est pratiquée dans certains cas (pression artérielle systolique basse, hypotension symptomatique, hyperkaliémie, altération de la fonction rénale, sujet âgé, insuffisance hépatique modérée), ainsi que, parfois, une adaptation posologique des traitements concomitants.

TEG1 Entresto ${ }^{\circledR}$ peut être administré pendant ou en dehors des repas, les comprimés devant être avalés avec un verre d'eau.

\section{T2 Contre-indications}

De nombreuses contre-indications sont mentionnées dans le résumé des caractéristiques du produit $(\mathrm{RCP})$ :

- hypersensibilité aux substances actives ou à l'un des excipients ;

- utilisation concomitante d'IEC (Entresto ${ }^{\circledR}$ ne doit être administré que 36 heures après l'arrêt de I'IEC) ;

- antécédent d'angiœdème lié à un traitement antérieur par IEC ou antagonistes des récepteurs de l'angiotensine II (ARAII) ;

- angiœdème héréditaire ou idiopathique ;

- insuffisance hépatique sévère, cirrhose biliaire ou cholestase ;

- deuxième et troisième trimestres de la grossesse.

\section{T2 Effets indésirables}

TEG1 Les effets indésirables le plus fréquemment rapportés ont été l'hypotension, l'hyperkaliémie et une altération de la fonction rénale.

TEG1 Des angiœdèmes peuvent survenir, une situation dans laquelle le traitement par Entresto ${ }^{\circledR}$ doit être arrêté immédiatement et ne pas être réadministré. Un traitement et une surveillance appropriés doivent être mis en place jusqu'à la disparition totale et durable des signes et symptômes.

\section{T1 Précautions et surveillance du traitement}


TEG1 Chez les patients ayant une kaliémie $>5,4 \mathrm{mmol} / \mathrm{L}$, le traitement ne doit pas être initié. La kaliémie doit être surveillée, en particulier chez les patients présentant des facteurs de risque (insuffisance rénale, diabète, hypoaldostéronisme) mais aussi chez ceux qui ont un régime alimentaire riche en potassium ou prennent des antagonistes du récepteur des minéralocorticoïdes. En cas d'hyperkaliémie cliniquement significative, une adaptation des traitements concomitants, une diminution de la posologie ou un arrêt sont recommandés.

TEG1 La pression artérielle doit être surveillée en routine lors de l'initiation du traitement ou de l'adaptation posologique. Le traitement ne doit pas être commencé tant que la pression artérielle systolique (PAS) n'est pas $\geq 100 \mathrm{mmHg}$. Une dose initiale de $24 \mathrm{mg} / 26 \mathrm{mg}$ deux fois par jour doit être envisagée chez les patients ayant une PAS comprise entre 100 et $110 \mathrm{mmHg}$.

TEG1 La fonction rénale des patients atteints d'insuffisance cardiaque doit toujours être évaluée, l'insuffisance rénale légère et modérée augmentant le risque de développer une hypotension. Par ailleurs, l'administration d'Entresto ${ }^{\circledR}$ peut être associée à une dégradation de la fonction rénale.

\section{T2 Interactions médicamenteuses}

TEG1 L'association avec les IEC est contre-indiquée en raison d'un risque accru d'angiœdème. Entresto ${ }^{\circledR}$ ne doit être initié que 36 heures après la prise de la dernière administration d'IEC. De même, en cas d'arrêt de traitement par Entresto ${ }^{\circledR}$, le traitement par IEC ne devra être commencé que 36 heures après la dernière prise de l'association sacubitril/valsartan.

TEG1 Un produit contenant un autre sartan que le valsartan ne doit pas être administré en association.

TEG1 En cas de co-administration avec des statines, une prudence particulière s'impose. Toutefois, aucune interaction médicamenteuse cliniquement significative n'a été observée lors de l'association avec la simvastatine.

TEG1 Avec les inhibiteurs de la phosphodiestérase de type 5 (PDE5), dont le sildénafil, un risque de baisse de la pression artérielle existe.

TEG1 L'utilisation concomitante de diurétiques antikaliurétiques (triamtérène, amiloride), d'antagonistes du récepteur des minéralocorticoïdes (par exemple, spironolactone et éplérénone), de suppléments potassiques, de substituts de sel contenant du potassium ou d'autres produits tels que l'héparine peut entraîner une augmentation de la kaliémie et une augmentation de la créatininémie. Une surveillance de la kaliémie est recommandée.

TEG1 Les patients qui prennent des anti-inflammatoires non stéroïdiens (AINS), dont les inhibiteurs de la cyclo-oxygénase $\mathbf{2}$ (COX-2), doivent bénéficier d'une surveillance de la fonction rénale au moment de l'instauration ou de la modification du traitement.

TEG1 L'association avec le lithium n'est pas recommandée en raison du risque d'augmentation de la lithémie. 
TEG1 L'administration concomitante d'Entresto ${ }^{\circledR}$ avec des inhibiteurs d'OATP1B1, d'OATP1B3 ou d'OAT3 (comme la rifampicine ou la ciclosporine), d'OAT1 (tels le ténofovir et le cidofovir) ou de MRP2 (comme le ritonavir) peut accroître l'exposition systémique du LBQ657 ou du valsartan. Une surveillance adaptée doit être instaurée lors de l'initiation ou de l'arrêt de traitement avec de tels médicaments.

Encadré

Entresto $^{\circledR}$, grossesse et allaitement

TEG1 Durant la grossesse, l'administration d'Entresto ${ }^{\circledR}$ n'est pas recommandée pendant le premier trimestre et elle est contre-indiquée pendant les deuxième et troisième trimestres.

TEG1 L'allaitement est déconseillé en raison du risque potentiel d'effets indésirables chez les nourrissons allaités.

Respecter cet emplacement pour l'encadré

T1 Une association de deux antiviraux pour l'hépatite C

TEG1 Spécialité : Maviret ${ }^{\circledR}$ (glécaprévir, pibrentasvir), laboratoire AbbVie.

TEG1 Présentation : comprimé pelliculé à $100 \mathrm{mg} / 40 \mathrm{mg}$ (boîte de 84).

TEG1 Prescription et délivrance : médicament en liste I. Prescription hospitalière réservée aux spécialistes en gastro-entérologie et hépatologie, en médecine interne ou en infectiologie.

TEG1 Remboursement Sécurité sociale à $100 \%$. Collectivités.

TEG1 ASMR : IV.

TEG1 Maviret ${ }^{\circledR}$ est indiqué dans le traitement de l'infection chronique par le virus de l'hépatite C (VHC) chez l'adulte.

TEG1 Maviret ${ }^{\circledR}$ est une association à dose fixe de deux antiviraux à action directe (AAD) pangénotypique :

- le glécaprévir, un inhibiteur de la protéase NS3/4A, nécessaire au clivage protéolytique de la polyprotéine codée du VHC (en formes matures des protéines NS3, NS4A, NS4B, NS5A et NS5B) et qui est essentielle à la réplication virale ;

- le pibrentasvir, un inhibiteur de la NS5A, protéine essentielle à la réplication de l'acide ribonucléique (ARN) viral et à l'assemblage des virions [5-8]. 
T2 Posologie

TEG1 La dose recommandée est de $\mathbf{3 0 0 ~} \mathbf{m g} / \mathbf{1 2 0} \mathbf{~ m g}$ (soit trois comprimés de $100 \mathrm{mg} / 40 \mathrm{mg}$ ) une fois par jour avec de la nourriture.

TEG1 Les durées du traitement varient de 8 à 16 semaines selon le génotype du virus en cause.

\section{T2 Contre-indications}

La prise de l'association glécaprévir/pibrentasvir est contre-indiquée en cas :

- d'hypersensibilité aux substances actives ou à l'un des excipients ;

- d'insuffisance hépatique sévère (Maviret ${ }^{\circledR}$ n'est pas recommandé chez les patients présentant une insuffisance hépatique modérée) ;

- de co-administration avec les produits contenant de l'atazanavir, avec l'atorvastatine, la simvastatine, le dabigatran étexilate, les médicaments contenant de l'éthinylestradiol, les inducteurs puissants de la glycoprotéine P (P-gp) et du cytochrome P450 3A (CYP3A) (rifampicine, carbamazépine, millepertuis, phénobarbital, phénytoïne et primidone, par exemple).

Ce médicament contenant du lactose, les patients présentant une intolérance au galactose, un déficit en lactase de Lapp ou un syndrome de malabsorption du glucose ou du galactose (maladies héréditaires rares) ne doivent pas le prendre.

\section{T2 Effets indésirables}

Les effets indésirables le plus fréquemment rapportés (incidence $10 \%$ ) au cours des essais cliniques ont été des céphalées et de la fatigue. Moins de 0,1\% des patients ont présenté des effets indésirables graves (accident ischémique transitoire).

\section{T2 Précautions et surveillance du traitement}

Un dépistage du virus de l'hépatite $B(\mathrm{VHB})$ doit être réalisé chez tous les patients avant l'initiation du traitement. En effet, les patients co-infectés par le VHB et le VHC sont exposés à un risque de réactivation du VHB et doivent, par conséquent, être surveillés et pris en charge.

\section{T2 Interactions médicamenteuses}

TEG1 Une administration concomitante avec les produits suivants est contre-indiquée : dabigatran, rifampicine, éthinylestradiol, millepertuis, atanazavir-ritonavir, atorvastatine et simvastatine. 
TEG1 Avec la digoxine, la prudence et une surveillance des concentrations thérapeutiques de la digoxine sont recommandées.

TEG1 L'utilisation concomitante avec les antiépileptiques (carbamazépine, phénytoïne, phénobarbital et promidone) peut entraîner une réduction de l'effet thérapeutique de Maviret ${ }^{\circledR}$.

TEG1 Avec les antirétroviraux, la co-administration n'est pas recommandée :

- darunavir-ritonavir ;

- éfavirenz-emtricitabine-ténofovir disoproxil (risque de réduction de l'effet thérapeutique de Maviret ${ }^{\oplus}$ ) ;

- lopinavir-ritonavir.

TEG1 Avec les statines, la prudence est conseillée :

- les doses de pravastatine et rosuvastatine ne doivent pas dépasser, respectivement, $20 \mathrm{mg}$ et $5 \mathrm{mg}$ par jour ;

- les interactions avec la fluvastatine et la pitavastatine sont probables ; l'administration d'une faible dose de statine est recommandée au début du traitement par AAD.

\section{TEG1 Avec les immunosuppresseurs :}

- chez les patients nécessitant des doses stables de ciclosporine $>100 \mathrm{mg}$ par jour, l'utilisation de Maviret ${ }^{\circledR}$ est déconseillée ; au cas où le traitement en association est inévitable, il peut être envisagé, sous surveillance clinique étroite, si le bénéfice l'emporte sur le risque ;

- la co-administration avec le tacrolimus appelle la prudence, une augmentation de l'exposition à ce médicament étant attendue. Par conséquent, un suivi thérapeutique pharmacologique du tacrolimus est recommandé ainsi qu'un ajustement de la posologie en conséquence.

TEG1 Avec les antivitamines K, une stricte surveillance de l'international normalized ratio (INR) est nécessaire en raison du risque de modification de la fonction hépatique.

Encadré

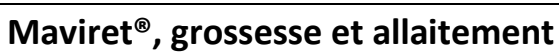

TEG1 Pendant la grossesse, l'utilisation de Maviret ${ }^{\circledR}$ n'est pas recommandée par mesure de précaution.

TEG1 Un risque pour l'enfant allaité ne peut être exclu. II doit être décidé d'arrêter l'allaitement ou $d^{\prime}$ interrompre/éviter le traitement par Maviret ${ }^{\circledR}$ en évaluant le bénéfice de l'allaitement pour l'enfant au regard de celui du traitement pour la mère. 
T1 Une association de trois antiviraux pour l'hépatite C

TEG1 Spécialité : Vosevi ${ }^{\circledR}$ (sofosbuvir, velpatasvir, voxilaprévir), laboratoire Gilead Sciences.

TEG1 Présentation : comprimé pelliculé à 400 mg/100 mg/100 mg (flacon de 28).

TEG1 Prescription et délivrance : médicament en liste I. Prescription hospitalière réservée aux spécialistes en gastro-entérologie et hépatologie, en médecine interne ou en infectiologie.

TEG1 Remboursement Sécurité sociale à $100 \%$. Collectivités.

TEG1 ASMR : IV.

TEG1 Vosevi ${ }^{\circledR}$, disponible à la fois en ville et en rétrocession, est indiqué pour le traitement de l'infection chronique par le VHC chez les adultes.

TEG1 Ce médicament une association fixe de trois antiviraux pangénotypiques :

- le sofosbuvir, un inhibiteur pangénotypique de I'ARN polymérase ARN-dépendante NS5B du VHC, nécessaire pour la réplication du virus ;

- le velpatasvir, un inhibiteur pangénotypique du VHC ciblant la protéine NS5A du VHC, nécessaire pour la réplication virale ;

- le voxilaprévir, un inhibiteur pangénotypique de la protéase NS3/4A du VHC [9-11].

\section{T2 Posologie}

TEG1 La dose recommandée est d'un comprimé une fois par jour, à prendre avec de la nourriture.

TEG1 Les durées de traitement varient de 8 à 12 semaines.

\section{T2 Contre-indications}

Une hypersensibilité aux substances actives ou à l'un des excipients contre-indique l'administration de Vosevi $^{\circledR}$.

Plusieurs co-administrations sont proscrites :

- avec de puissants inducteurs de la P-gp et/ou de puissants inducteurs du CYP450 (par exemple, rifampicine, rifabutine, millepertuis, carbamazépine, phénobarbital et phénytoïne) ; 
- la rosuvastatine ou le dabigatran étexilate ;

- des médicaments contenant de l'éthinylestradiol comme les contraceptifs oraux combinés ou les anneaux vaginaux contraceptifs.

TEG1 Vosevi ${ }^{\circledast}$ contenant du lactose, les patients présentant des troubles héréditaires rares d'intolérance au galactose, de déficit en lactase de Lapp, ou de malabsorption du glucose-galactose ne doivent pas prendre ce médicament.

\section{T2 Effets indésirables}

TEG1 Les effets indésirables très fréquents sont des maux de tête, des diarrhées et des nausées.

TEG1 Les effets couramment observés sont : douleurs abdominales, baisse de l'appétit, vomissements, myalgies et augmentation de bilirubine.

TEG1 Des effets sont survenus plus rarement : rash et spasmes musculaires.

\section{T2 Précautions et surveillance du traitement}

TEG1 L'amiodarone ne doit être administrée chez les patients prenant Vosevi ${ }^{\circledR}$ qu'en cas d'intolérance ou de contre-indication aux autres traitements anti-arythmiques. Si son utilisation concomitante est jugée nécessaire, un suivi étroit doit être mis en place lors de l'initiation du traitement par Vosevi ${ }^{\circledast}$. Compte tenu de la longue demi-vie de l'amiodarone, une surveillance appropriée est nécessaire chez les patients ayant arrêté sa prise au cours des derniers mois et devant débuter un traitement par Vosevi ${ }^{\circledR}$. Tous les patients traités par Vosevi ${ }^{\circledR}$ et recevant de l'amiodarone avec ou sans autres médicaments bradycardisants doivent également être avertis des symptômes de bradycardie et de troubles de la conduction, dont la survenue doit les conduire à consulter un médecin.

TEG1 Le dépistage du VHB doit être effectué chez tous les patients avant le début du traitement. Les patients co-infectés par le VHC/VHB sont à risque de réactivation du VHB et doivent donc être surveillés et pris en charge conformément aux recommandations cliniques en vigueur.

TEG1 En cas d'insuffisance hépatique, aucun ajustement de la dose de Vosevi ${ }^{\circledast}$ n'est nécessaire. Toutefois, ce médicament n'est pas recommandé chez les patients présentant une insuffisance hépatique modérée ou sévère. 
TEG1 De très nombreuses interactions médicamenteuses existent pour ce produit. Lors de sa dispensation il est donc important d'analyser soigneusement les interactions potentielles avec les autres médicaments pris par le patient, y compris ceux relevant de l'automédication.

TEG1 Avec les réducteurs d'acidité : il est conseillé de respecter un intervalle de quatre heures entre la prise d'un antiacide et celle de Vosevi ${ }^{\circledR}$; les antagonistes des récepteurs $\mathrm{H}_{2}$ peuvent être administrés en même temps ou à distance, à une dose ne dépassant pas l'équivalent de $40 \mathrm{mg}$ de famotidine deux fois par jour ; les inhibiteurs de la pompe à protons (IPP) peuvent être administrés à des doses maximales comparables à $20 \mathrm{mg}$ d'oméprazole.

TEG1 L'amiodarone ne peut être utilisée que si aucun traitement alternatif n'est disponible. Une surveillance étroite est recommandée en cas de co-administration.

TEG1 La co-administration avec la digoxine peut augmenter la concentration de cette dernière dont il est recommandé de surveiller la concentration thérapeutique.

TEG1 Avec les anticoagulants : Vosevi ${ }^{\circledR}$ est contre-indiqué avec le dabigatran et sa co-administration avec l'édoxaban n'est pas recommandée. Si l'utilisation d'un inhibiteur direct du facteur Xa est jugée nécessaire, l'apixaban ou le rivaroxaban peuvent être envisagés. Les valeurs de l'INR doivent être surveillées étroitement en cas d'administration concomitante avec tous les antagonistes de la vitamine $\mathrm{K}$.

TEG1 L'association avec les anticonvulsivants, la phénytoïne, le phénobarbital et la carbamazépine, est proscrite.

TEG1 Concernant les antimycobactériens, la prise de Vosevi ${ }^{\circledR}$ est contre-indiquée avec la rifabutine et sa co-administration avec la rifapentine n'est pas recommandée.

TEG1 La co-administration avec certains antirétroviraux, l'éfavirenz, l'emtricitabine, le fumarate de ténofovir disoproxil, l’atazanavir et le lopinavir, est déconseillée.

TEG1 L’association avec le millepertuis est contre-indiquée.

TEG1 Avec les inhibiteurs de la HMG-CoA réductase, l'association n'est en général pas recommandée. Celle avec la rosuvastatine est contre-indiquée et celle avec la pravastatine à une dose ne dépassant pas $40 \mathrm{mg}$ peut être envisagée.

TEG1 La co-administration avec la ciclosporine est proscrite.

TEG1 Avec les contraceptifs oraux contenant de l'éthinylestradiol, la prise de Vosevi ${ }^{\circledR}$ est contreindiquée. Des méthodes alternatives (avec progestatif seul ou non hormonales, par exemple) doivent être envisagées.

TEG1 Avec le modafinil, la co-administration n'est pas recommandée. 
Vosevi $^{\circledR}$, grossesse et allaitement $^{2}$

TEG1 Pendant la grossesse, l'administration de Vosevi ${ }^{\circledR}$ n'est pas recommandée par mesure de précaution.

TEG1 L'allaitement est proscrit.

Respecter cet emplacement pour l'encadré / sur 1 colonne

T1 Une trithérapie pour le sida

TEG1 Spécialité : Biktarvy ${ }^{\circledR}$ (bictégravir, emtricitabine, ténofovir alafénamide), laboratoire Gilead Sciences.

TEG1 Présentation : comprimé pelliculé à 50 mg/200 mg/25 mg (flacon de 30).

TEG1 Prescription et délivrance : médicament en liste I. Prescription initiale hospitalière annuelle. Renouvellement non restreint. Inscrit sur la liste de rétrocession, disponible en ville comme à l'hôpital.

TEG1 Remboursement Sécurité sociale à $100 \%$. Collectivités.

TEG1 ASMR : V.

TEG1 Biktarvy ${ }^{\circledast}$ est indiqué pour le traitement des adultes infectés par le virus du sida (VIH-1) sans preuve actuelle ou antérieure de résistance à la classe des inhibiteurs de l'intégrase, à l'emtricitabine ou au ténofovir.

TEG1 Ce médicament associe trois principes actifs qui agissent sur différentes enzymes du cycle de réplication du virus du sida :

- le bictégravir, un inhibiteur de l'intégrase (INI) ;

- I'emtricitabine, un inhibiteur nucléosidique de la transcriptase inverse (INTI) ;

- le ténofovir alafénamide, un inhibiteur nucléotidique de la transcriptase inverse (INtTI) [12-14].

\section{T2 Posologie}

La posologie est d'un comprimé au long cours une fois par jour, avec ou sans nourriture. Ce dernier ne doit pas être croqué, écrasé ou coupé. 
Une hypersensibilité aux substances actives ou à l'un des excipients contre-indique la prise de Biktarvy ${ }^{\circledR}$ qui ne doit, par ailleurs, pas être administré avec la rifampicine et le millepertuis.

Ce traitement n'est pas recommandé chez les patients présentant une clairance de la créatinine inférieure à $30 \mathrm{~mL} / \mathrm{min}$ ou présentant une insuffisance hépatique sévère.

\section{T2 Effets indésirables}

TEG1 Les effets indésirables le plus fréquemment rapportés au cours des études cliniques ont été des céphalées, des diarrhées et des nausées. De nombreux autres effets peuvent survenir : dépression, vertiges, fatigue...

TEG1 Une augmentation du poids corporel ainsi que des taux de lipides et de glucose sanguin peuvent être observés.

TEG1 Un risque potentiel de néphrotoxicité ne peut être exclu.

\section{T2 Précautions et surveillance du traitement}

TEG1 Chez les patients présentant un déficit immunitaire sévère au moment de l'instauration du traitement antirétroviral, une réaction inflammatoire à des infections opportunistes asymptomatiques ou résiduelles peut se produire et entraîner des manifestations cliniques graves ou une aggravation des symptômes. Ces réactions s'observent au cours des premières semaines ou des premiers mois de traitement. Tout symptôme inflammatoire doit donc être évalué et traité si nécessaire.

TEG1 En prévention de l'ostéonécrose, il est conseillé aux patients de consulter un médecin s'ils éprouvent des douleurs et des arthralgies, une raideur articulaire ou des difficultés pour se mouvoir.

\section{T2 Interactions médicamenteuses}

TEG1 Biktarvy ${ }^{\circledR}$ ne doit pas être co-administré à jeun simultanément avec des médicaments antiacides contenant du magnésium/de l'aluminium ou des compléments à base de fer :

- au moins deux heures avant ou avec de la nourriture deux heures après les antiacides contenant du magnésium et/ou de l'aluminium ;

- au moins deux heures avant la prise de compléments à base de fer ou conjointement avec de la nourriture. 
TEG1 La co-administration avec certains médicaments n'est pas recommandée : atazanavir, bocéprevir, carbamazépine, oxcarbazépine, phénobarbital, phénytoïne, rifabutine, rifapentine, ciclosporine ou sucralfate.

TEG1 Diverses autres associations nécessitent des précautions : macrolides, ciclosporine, vérapamil, dronédarone, glécaprévir/pibrentasvir...

TEG1 Compte tenu du nombre important d'interactions médicamenteuses potentielles, il est nécessaire de consulter le RCP pour en obtenir une liste exhaustive.

\section{Encadré}

\section{Biktarvy ${ }^{\circledR}$, grossesse et allaitement}

TEG1 Lors de la grossesse, Biktarvy ${ }^{\circledR}$ ne doit être utilisé que si les bénéfices potentiels du traitement sont supérieurs aux risques potentiels pour le fœtus.

TEG1 L'allaitement est déconseillé aux femmes infectées par le virus de l'immunodéficience humaine, quelles que soient les circonstances, afin d'éviter la transmission du virus au nourrisson.

Respecter cet emplacement pour l'encadré / sur 1 colonne

T1 Un inhibiteur de l'IL-17 pour le psoriasis

TEG1 Spécialité : Kyntheum ${ }^{\circledR}$ (brodalumab), laboratoire Leo Pharma.

TEG1 Présentation : solution injectable à $210 \mathrm{mg}$ en seringue préremplie de $1,5 \mathrm{~mL}$ munie d'une aiguille $27 \mathrm{G}$ d'une longueur $1 / 2$ po (boîte de 2 ).

TEG1 Prescription et déliurance : médicament en liste I. Prescription initiale hospitalière. Prescription initiale et renouvellement réservés aux spécialistes en dermatologie ou en médecine interne. Médicament d'exception.

TEG1 Remboursement Sécurité sociale à $65 \%$. Collectivités.

TEG1 ASMR : V.

TEG1 Kyntheum ${ }^{\circledast}$ est indiqué dans le traitement du psoriasis en plaques modéré à sévère chez l'adulte nécessitant un traitement systémique [15-17].

TEG1 Le brodalumab est un immunosuppresseur, inhibiteur de l'IL-17. Cet anticorps monoclonal recombinant entièrement humain se lie avec une affinité élevée à I'IL-17RA humaine et bloque l'activité biologique des cytokines pro-inflammatoires, ce qui entraîne l'inhibition de l'inflammation et des symptômes cliniques associés au psoriasis. En effet, une augmentation des concentrations de 
cytokines de la famille IL-17 en cas de psoriasis a été rapportée. La neutralisation de l'IL-17RA inhibe les réponses induites par les cytokines IL-17, ce qui normalise l'inflammation cutanée.

Buxer_illus2

\section{T2 Posologie}

TEG1 La dose recommandée est de $\mathbf{2 1 0} \mathbf{m g}$ en injection sous-cutanée (SC), administrée aux semaines 0,1 et 2 , suivie de $210 \mathrm{mg}$ toutes les deux semaines.

TEG1 Après une formation adaptée à la technique d'injection SC, les patients peuvent s'administrer eux-mêmes Kyntheum ${ }^{\circledR}$ si un médecin estime que cela est approprié. Ils doivent être informés de la nécessité d'injecter tout le volume de la seringue, qui ne doit pas être agitée, conformément aux instructions détaillées figurant dans la notice. Chaque seringue préremplie est à usage unique. Le produit ne doit pas être injecté dans des zones où la peau est sensible au toucher, rouge, indurée, épaissie, écailleuse, présente un hématome ou est atteinte de psoriasis.

TEG1 Compte tenu du risque potentiel important de réactions d'hypersensibilité avec le brodalumab administré par voie SC mentionné dans le plan de gestion des risques (PGR) de Kyntheum $^{\circledR}$, la Commission de la transparence conseille, comme pour les autres traitements biologiques, de réaliser la première injection dans une structure de soins adaptée.

\section{T2 Durée du traitement}

En l'absence de réponse après 12 à 16 semaines de traitement, son arrêt doit être envisagé. Certains patients présentant une réponse initiale partielle peuvent obtenir une amélioration en poursuivant son administration au-delà de 16 semaines.

\section{T2 Contre-indications}

Kyntheum ${ }^{\circledast}$ est contre-indiquée en cas :

- d'hypersensibilité à la substance active ou à l'un des excipients ;

- de maladie de Crohn active ;

- d'infections évolutives et cliniquement significatives (comme une tuberculose active). 
TEG1 Les effets indésirables le plus fréquemment rapportés chez tous les patients traités par Kyntheum ${ }^{\circledR}$ ont été des arthralgies, des céphalées, de la fatigue, des diarrhées et des douleurs oropharyngées.

TEG1 Compte tenu de l'effet immunosuppresseur de ce médicament, il convient d'être vigilant quant au risque potentiel infectieux. Quelques cas de neutropénies ont été rapportés, mais aucune infection grave n'y a été associée.

Encadré

Kyntheum $^{\circledR}$, femme en âge de procréer, grossesse et allaitement

TEG1 Les femmes en âge de procréer doivent utiliser une méthode de contraception efficace pendant le traitement et au moins douze semaines après son arrêt.

TEG1 Pendant la grossesse, l'utilisation de Kyntheum ${ }^{\circledR}$ doit être évitée par mesure de précaution.

TEG1 La décision d'interrompre l'allaitement ou d'interrompre/de s'abstenir du traitement par Kyntheum ${ }^{\circledR}$ doit être prise en prenant en compte le bénéfice de l'allaitement pour l'enfant par rapport au bénéfice du traitement pour la femme.

Respecter cet emplacement pour l'encadré/ sur 1 colonne

\section{T2 Précautions et surveillance du traitement}

TEG1 Les patients ayant des antécédents de maladie de Crohn doivent être suivis afin de détecter tous les signes et symptômes d'une forme évolutive. Le cas échéant, le traitement doit être définitivement arrêté.

TEG1 Des comportements et idées suicidaires, ainsi que des passages à l'acte, ont été rapportés chez des patients traités par Kyntheum ${ }^{\circledR}$. Les risques et les bénéfices du traitement doivent être évalués attentivement chez les sujets présentant des antécédents de dépression et/ou de comportements ou d'idées suicidaires, ainsi que chez ceux qui développent ce type de symptômes. Les patients, soignants et proches doivent être informés de la nécessité d'être attentifs à l'apparition ou à l'exacerbation d'une dépression, d'idées suicidaires, d'anxiété ou d'autres changements d'humeur, et de contacter leur professionnel de santé le cas échéant. Si un patient présente de nouveaux symptômes de dépression et/ou des idées ou des comportements suicidaires, ou une aggravation de ceux-ci, il est recommandé d'arrêter le traitement par Kyntheum ${ }^{\circledR}$.

TEG1 Le risque d'infections peut être augmenté. Il convient d'informer les personnes sous traitement de la nécessité de consulter un médecin en cas d'apparition de signes ou de symptômes évocateurs. Kyntheum ${ }^{\circledR}$ ne doit pas être administré aux patients présentant une tuberculose active. 
TEG1 Il est recommandé de s'assurer que les patients sont à jour de leurs vaccinations, conformément aux recommandations vaccinales locales, avant l'instauration d'un traitement par Kyntheum ${ }^{\circledast}$.

\section{T2 Interactions médicamenteuses}

Les vaccins vivants ne doivent pas être administrés en même temps que Kyntheum ${ }^{\circledR}$.

Note

${ }^{1}$ Les peptides natriurétiques exercent leurs effets physiologiques en activant des récepteurs membranaires à activité guanylate-cyclase, entraînant une augmentation des concentrations du second messager hormonal, la guanosine monophosphate cyclique (GMPc).

\section{Références}

[1] Haute Autorité de santé (HAS). Synthèse d'avis de la Commission de la transparence. Entresto (sacubitril/valsartan), antagoniste de l'angiotensine II en association à un inhibiteur de l'endopeptidase neutre. Mai 2016. www.has-sante.fr/portail/upload/docs/application/pdf/201609/entresto_synthese_ct14835.pdf

[2] Haute Autorité de santé (HAS). Commission de la transparence. Avis du 11 mai 2016. Sacubitril/valsartan. www.has-sante.fr/portail/upload/docs/evamed/CT14835_ENTRESTO_PIC_INS_Avis3_CT14835.pdf

[3] Paitraud D. Entresto (sacubitril, valsartan) : nouvelle association fixe dans le traitement de I'insuffisance cardiaque chronique. Actualités Vidal, 2 février 2016.

www.vidal.fr/actualites/19041/entresto_sacubitril_valsartan_nouvelle_association_fixe_dans_le_tra itement_de_I_insuffisance_cardiaque_chronique/

[4] Paitraud D. Insuffisance cardiaque chronique : Entresto (valsartan/sacubitril) désormais disponible en ville. Actualités Vidal, 17 octobre 2018.

www.vidal.fr/actualites/22910/insuffisance_cardiaque_chronique_entresto_valsartan_sacubitril_de sormais_disponible_en_ville/

[5] Haute Autorité de santé (HAS). Synthèse d'avis de la Commission de la transparence. Maviret (glécaprévir/pibrentasvir), association fixe d'antiviraux à action directe. Décembre 2017. www.hassante.fr/portail/upload/docs/application/pdf/2018-07/maviret_synthese_ct16434.pdf 
[6] Haute Autorité de santé (HAS). Commission de la transparence. Avis du 6 décembre 2017. Glécaprévir/pibrentasvir. www.has-sante.fr/portail/upload/docs/evamed/CT16434_MAVIRET_PIC_INS_Avis2_CT16434.pdf

[7] Paitraud D. Hépatite C. Arrivée de Maviret en ville, le premier antiviral d'action directe pour le traitement de l'hépatite C. Pharma radio, 21 mars 2018. www.pharmaradio.fr/article/arrivee-demaviret-en-ville-le-premier-antiviral-d-action-directe-pour-le-traitement-de-I-hepatite-c- 80

[8] Paitraud D. Hépatite C chronique : Maviret, première association d'antiviraux d'action directe disponible en ville. Actualités Vidal, 14 mars 2018.

www.vidal.fr/actualites/22612/hepatite_c_chronique_maviret_premiere_association_d_antiviraux_ d_action_directe_disponible_en_ville/

[9] Haute Autorité de santé (HAS). Synthèse d'avis de la Commission de la transparence. Vosevi (sofosbuvir / velpatasvir / voxilaprévir), association fixe d'antiviraux à action directe.

Décembre 2017. www.has-sante.fr/portail/upload/docs/application/pdf/2018-

06/vosevi_sapub_ct16443.pdf

[10] Haute Autorité de santé (HAS). Commission de la transparence. Avis du 6 décembre 2017. Sofosbuvir / velpatasvir / voxilaprévir. www.has-sante.fr/portail/upload/docs/evamed/CT16443_VOSEVI_PIC_INS_Avis2_CT16443.pdf

[11] Paitraud D. Hépatite C chronique : Vosevi, nouvel antiviral d'action directe disponible en ville. Actualités Vidal, 4 avril 2018.

www.vidal.fr/actualites/22646/hepatite_c_chronique_vosevi_nouvel_antiviral_d_action_directe_dis ponible_en_ville/

[12] Cochois I. Biktarvy (bictégravir, emtricitabine, ténofovir alafénamide) : nouvelle trithérapie contre l'infection par le VIH-1. Actualités Vidal, 23 novembre 2018.

www.vidal.fr/actualites/22965/biktarvy_bictegravir_emtricitabine_tenofovir_alafenamide_nouvelle _tritherapie_contre_I_infection_par_le_vih_1

[13] Haute Autorité de santé (HAS). Avis de la Commission de la transparence du 13 décembre 2017. bictégravir, emtricitabine, ténofovir alafénamide. www.has-

sante.fr/portail/upload/docs/evamed/CT-17014_BIKTARVY_PIC_AVIS2_CT17014.pdf

[14] Haute Autorité de santé (HAS). Synthèse d'avis de la Commission de la transparence. Biktarvy (bictégravir, emtricitabine, ténofovir alafénamide), association d'antirétroviraux. Septembre 2018. www.has-sante.fr/portail/upload/docs/application/pdf/2018-12/biktarvy_sapub_ct17014.pdf

[15] Haute Autorité de santé (HAS). Synthèse d'avis de la Commission de la transparence.

Kyntheum (brodalumab), immunosuppresseur inhibiteur d'interleukines. Avril 2018. www.hassante.fr/portail/upload/docs/application/pdf/2018-07/kyntheum_synthese_ct16707.pdf

[16] Haute Autorité de santé (HAS). Commission de la transparence. Avis du 4 avril 2018.

Brodalumab. www.has-sante.fr/portail/upload/docs/evamed/CT-

16707_KYNTHEUM_PIS_INS_Avis3_CT16707.pdf 
[17] Paitraud D. Psoriasis en plaques : Kyntheum (brodalumab), nouvel inhibiteur de la voie de l'IL17. Actualités Vidal, 11 octobre 2018.

www.vidal.fr/actualites/22888/psoriasis_en_plaques_kyntheum_brodalumab_nouvel_inhibiteur_de _la_voie_de_I_il_17/

\section{Illustrations}

\section{Buxer_illus1}

(C) adragan/stock.adobe.com

Entresto ${ }^{\circledR}$ possède un double mécanisme d'action grâce à ses deux composés et indiqué dans le traitement de l'insuffisance cardiaque chronique symptomatique à fraction d'éjection réduite chez les patients adultes.

\section{En haut à gauche en deuxième page}

\section{Buxer_illus2}

(C) balisnake/stock.adobe.com

Immunosuppresseur inhibiteur de l'IL-17, le brodalumab est un anticorps monoclonal recombinant qui se lie à I'IL-17RA humaine et bloque l'activité biologique des cytokines pro-inflammatoires responsables du psoriasis.

\section{En haut à gauche près de son appel}




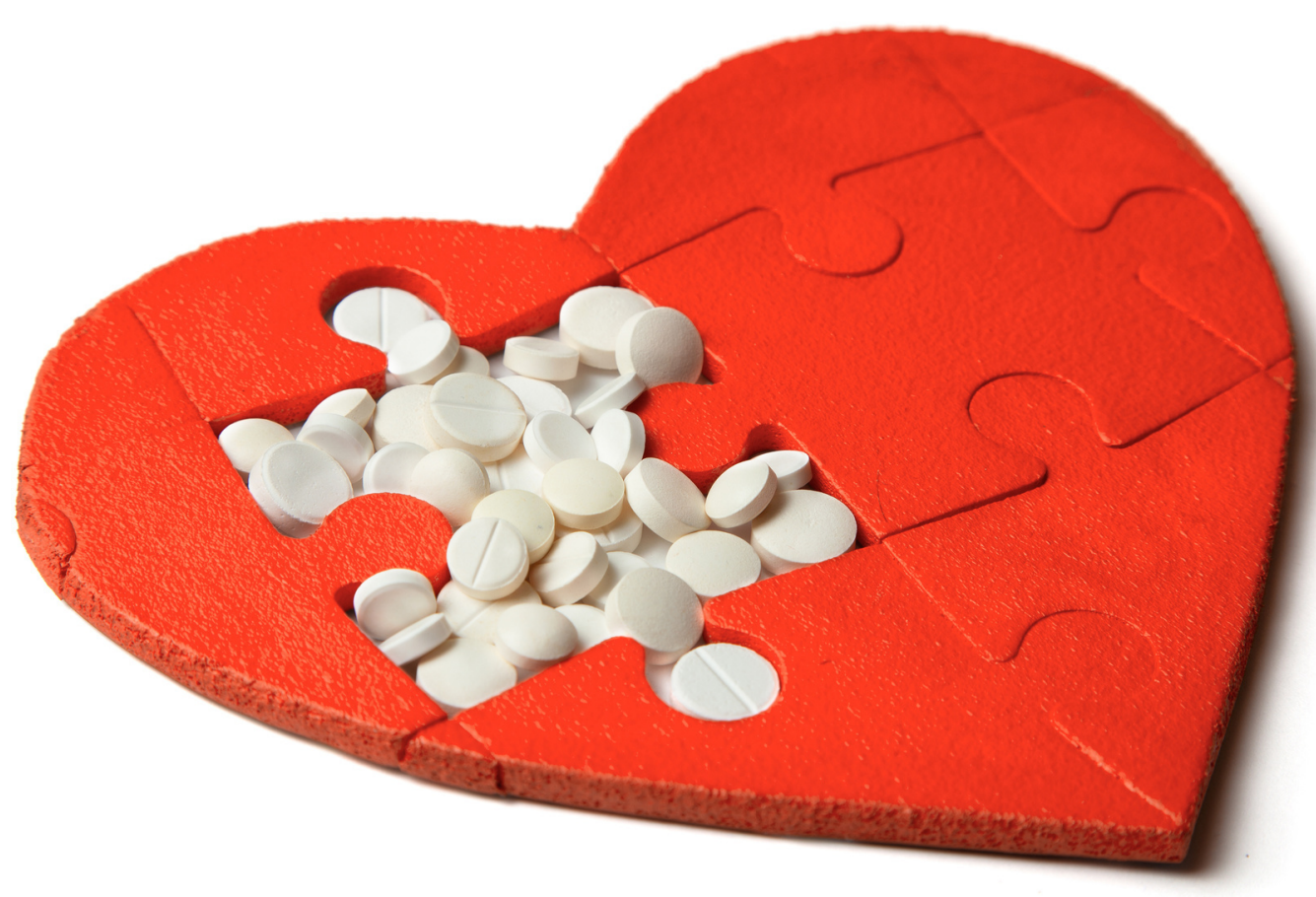


